\title{
Clinical significance of PD-L1 protein expression on tumor-associated macrophages in lung cancer
}

\author{
Kurt Alex Schalper ${ }^{1 *}$, Daniel Carvajal-Hausdorf ${ }^{1}$, Joseph McLaughlin ${ }^{1}$, Vamsidhar Velcheti ${ }^{2}$, Lieping Chen ${ }^{1}$, \\ Miguel Sanmamed ${ }^{1}$, Roy S Herbst ${ }^{3}$, David L Rimm¹ \\ From 30th Annual Meeting and Associated Programs of the Society for Immunotherapy of Cancer (SITC 2015) \\ National Harbor, MD, USA. 4-8 November 2015
}

\section{Introduction}

Tumor PD-L1 expression is associated with increased tumor-infiltrating lymphocytes (TILs) in diverse solid tumors, including lung cancer. In addition, PD-L1 upregulation in tumor and/or stromal immune cells has been associated with increased clinical benefit to PD-1/ PD-L1 axis blockers. The significance of PD-L1 expression in different immune cell subpopulations at the tumor microenvironment remains poorly understood. Here, we measured PD-L1 protein specifically in tumorassociated macrophages using objective methods and analyzed its clinical significance in human lung cancer.

\section{Methods}

Using multiplexed quantitative immunofluorescence (QIF) we measured the levels of PD-L1 protein (clone-E1L3N, Cell Signaling technology) in 554 stages I-IV FFPE lung carcinomas represented in two tissue-microarrays, one from Yale University [YTMA79, $\mathrm{n}=204$ ] and one from Greece [YTMA140, n=350]. PD-L1 was measured using multispectral QIF in CD68-positive cells (macrophages, clone-KP1, DAKO) or in tumor cells (clone-AE1/AE3, DAKO) based on fluorescence co-localization. The levels of CD3 (T cells, clone-E272, Novus), CD8 (cytotoxic $\mathrm{T}$ cells, clone-C8/144B, DAKO) and CD20 (B-lymphoctes, clone-L26, DAKO) were also determined in the tumor samples. Associations between the macrophage and tumor PD-L1 levels, clinico-pathological variables and survival were analyzed.

\section{Results}

In lung cancer samples, PD-L1 was detected in both macrophages and tumor cells with a predominant

\footnotetext{
${ }^{1}$ Yale University, New Haven, CT, USA

Full list of author information is available at the end of the article
}

membranous staining pattern. Overall, the levels of PDL1 in the CD68+ population were lower than in tumor cells in both cohorts $(834 \pm 314$ AU vs $1113 \pm 427 \mathrm{AU}$, in the Yale cohort, $\mathrm{P}<0.001$; and $895 \pm 451 \mathrm{AU}$ vs $1069 \pm 542 \mathrm{AU}$ in the Greek set, $\mathrm{P}<0.001)$. In both collections, the macrophage and tumor PD-L1 signal showed a positive non-linear association (Linear regression coefficient $\left[R^{2}\right]=0.311$ in the Yale cohort and $\mathrm{R}^{2}=0.43$ in the Greek collection). Using the median score as cutpoint, elevated macrophage PD-L1 content was significantly associated with high CD8+ and CD20+ cells in both cohorts. In addition, increased macrophage PD-L1 signal was marginally associated with longer overall survival in the Yale set $(\mathrm{HR}=0.676$ [CI:0.4501.081], $\mathrm{P}=0.05)$, but not in the Greek group $(\mathrm{HR}=0.790$ [CI:0.560-1.109], $\mathrm{P}=0.17)$.

\section{Conclusion}

PD-L1 is expressed in tumor-associated macrophages of human lung carcinomas. The macrophage PD-L1 signal is lower than in tumor epithelial cells and they show a positive association. Elevated macrophage PD-L1 is prominently associated with increased cytotoxic $\mathrm{T}$ lymphocytes and B cell infiltrates in lung tumors, but has limited prognostic value. Further studies will be required to evaluate the possible predictive role of PD-L1 expression in macrophages and other immune cell types.

\section{Authors' details \\ ${ }^{1}$ Yale University, New Haven, CT, USA. ${ }^{2}$ Cleveland Clinic, Cleveland, OH, USA. ${ }^{3}$ Yale Cancer Center, Yale School of Medicine, Yale University, New Haven, $C T$, USA.}

\section{Published: 4 November 2015}

doi:10.1186/2051-1426-3-S2-P415

Cite this article as: Schalper et al:: Clinical significance of PD-L1 protein expression on tumor-associated macrophages in lung cancer. Journal for ImmunoTherapy of Cancer 2015 3(Suppl 2):P415. 